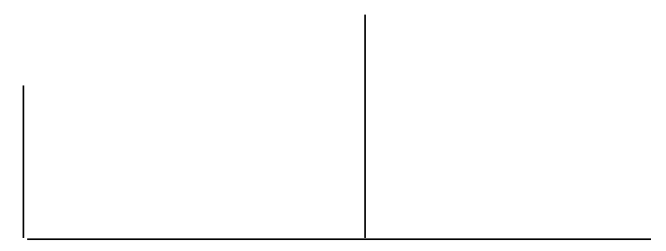

Rev. Latinoam. Psicopat. Fund., VI, 4, 54-67

\title{
La gratitud: una cualidad natural
}

José de Jesús Gonzáles Nunes

Maria del Pilar Rodríguez Cortés

\begin{abstract}
En este artículo se conceptúa la gratitud como un afecto cuya definición es la capacidad del individuo de agradecer, de poder reconocer los dones recibidos por un bienhechor. Se discute la epigénesis y evolución de la gratitud junto con otros procesos psíquicos como gratitud y defensas, gratitud y sentimientos de culpa, gratitud y otras cualidades humanas, gratitud y envidia, para concluir con cúal es la función de la gratitud en el proceso de psicoterapia psicoanalítca.
\end{abstract}

Palabras clave: Gratitud, defensas, sentimientos de culpa, envidia 


\section{Concepto de gratitud}

La gratitud es la capacidad que el individuo posee de poder agradecer, de poder reconocer los dones recibidos por un bienhechor. En la teoría clásica del psicoanálisis, este concepto adquiere sentido en la medida que se afirma, de acuerdo a las posturas pasivo-activo del actuar, que aquello que se vivió pasivamente durante la infancia, durante la vida adulta se repite activamente. Se vive pasivamente en la infancia porque el niño es un ser en desarrollo que no puede todavía vivir activamente todo lo que recibe pasivamente.

Si el niño recibió satisfacciones, placer, cuidados, respeto como persona, después lo repetirá activamente en forma de gratitud.

Dentro de la corriente psicoanalítica inglesa, insertada en la postura de relaciones de objeto, Klein (1957) explica la gratitud como la capacidad de reparar al objeto amado hacia el cual en forma inconsciente se dañó, agredió, maltrató y en la fantasía hasta se le mató.

En ambas posturas se nos muestra la gratitud como una capacidad de agradecimiento reactiva: en la primera es producto de una postura pasiva que reactivamente se vuelve activa, en la segunda es la capacidad reparadora producto de una tendencia destructiva. Sin embargo debemos considerar que en esa relación de objeto que se establece inicialmente con la madre, luego con el padre y hermanos si es que los hay; la madre, el padre y demás objetos significativos para el niño, le dan, le satisfacen sus necesidades e impulsos, dones que es casi imposible que el niño lo deje de reconocer, por lo tanto también existe una tendencia natural a expresar gratitud con el objeto dador, satisfactor o gratificante.

Si bien es cierto que el primer sentimiento, el primer pensamiento y la primera conducta reparadora se deben a la primera 


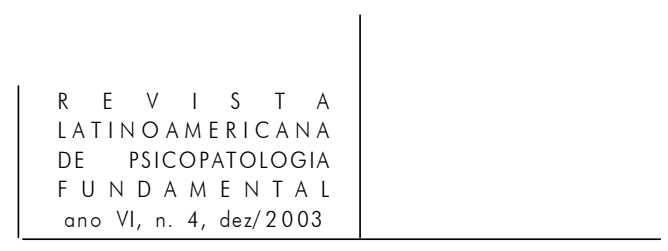

frustración, y son dolorosos (Rapaport, 1967), también es cierto que la primera satisfacción es gozosa y produce en la proyección deseos de que el objeto esté también satisfecho así como lo está el sujeto.

El fenómeno introyectivo-proyectivo también nos da cuenta de una gratitud natural, así como se proyectan envidias, dolores, temores, carencias, agresiones, fantasías destructivas etc., también se proyectan cualidades como el gozo, la seguridad, la prosperidad, la tranquilidad, el consuelo, la satisfacción; la gratitud.

\section{Epigénesis de la gratitud}

\section{a) Las protofantasías}

Entendemos la constitución de la personalidad en términos de tres factores fundamentales: constitucionales, del desarrollo y medio-ambientales.

Dentro de los factores constitucionales se incluyen los biológicos: herencia, constitución somática, umbrales de reacción etc. y los psicológicos. Dentro de los factores constitucionales psicológicos se encuentran la inteligencia con su componente somático, la memoria, las emociones, las fantasías y las protofantasías.

Las protofantasías son fantasías con contenidos humanos ancestrales, que se heredan de generación en generación, que bien equivalen a los arquetipos de Jung. Son postulados teóricos de difícil demostración empírica solo comprobables por sus manifestaciones inconscientes. Por lo tanto estamos hablando de una protofantasía de la gratitud.

Desde siempre el hombre nace inmerso en una religión o cuerpo de creencias en las que se supone existen deidades buenas y malas. Las deidades buenas siempre están disponibles para dar, proteger, amparar a los hombres y ayudarles a satisfacer sus necesidades y también ayudarles a resolver sus temores. En esta suposición teórica se inserta la idea de que la gratitud también es una protofantasía que consiste en agradecer a esos poderosos protectores el haberse desprendido de algo, poseer disponibilidad y dar sus dones por lo cual se les agradece y se les tiene gratitud.

\section{b) Mundo interno: incorporación-proyección}

Desde el nacimiento existe un yo primitivo y rudimentario, capaz de establecer relaciones objetales mediante los mecanismos de introyecciónproyección, manteniendo una gran fluidez en sus fronteras. 


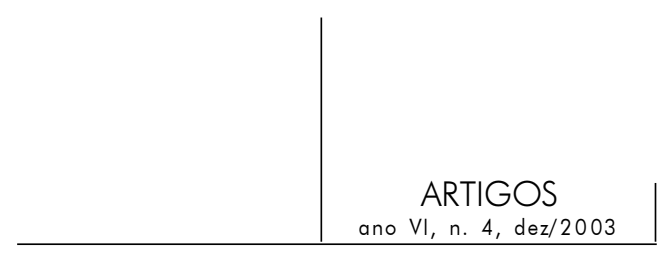

Las fantasías (Klein, 1955) son la vida del mundo interior y también son consideradas como una función destinada a establecer relaciones con los objetos del exterior ya sean estos buenos y malos. La fantasía inconsciente es el derivado de los impulsos y por consiguiente coexiste con los objetos desde el comienzo de la vida (Segal, 1992). En la psique del niño se vive al instinto vinculado con la fantasía de un objeto adecuado a él. En tal forma que cada impulso tiene una fantasía correspondiente.

Igualmente, con el paso del tiempo, se corrigió la teoría de que la libido era buscadora de placer por la teoría clínica de que la libido o los impulsos en general son buscadores de objetos (Fairbairn, 1978). En esta búsqueda de objetos el niño desea incorporar lo bueno del objeto y hacerlo parte de sí, y rechazar todo lo malo. Aunque generalmente esta situación no se da por la incapacidad del niño y porque la madre no siempre ofrece lo bueno. Al final el propósito del niño es intentar adquirir y guardar dentro de si al objeto ideal capaz de satisfacerlo siempre (Segal, 1992) e identificarse con él, una vez identificado con el objeto bueno puede mantener fuera al objeto malo, puesto que el objeto bueno, con su bondad, lo protege.

A medida que el infante internaliza el objeto bueno y se identifica parcialmente con él, se fortalece su yo. El crecimiento y fortalecimiento del yo se deben en parte al crecimiento fisiológico, pero también a la internalización de las experiencias buenas que permiten el crecimiento psicológico. Conforme el niño se fortalece siente menos atracción por proyectar lo malo hacia el exterior. En la medida que se siente más fuerte y menos amenazado y puede tolerar la tensión interna. Se establece un círculo benevolente, conforme sea más fuerte el yo del niño, menos se siente amenazado y proyecta menos. Y a medida que proyecta menos, sus objetos se vuelven menos amenazantes. Se reconoce que el poder sentirse deprimido cuando se aleja el objeto amado es un sentimiento normal. Es anormal el no poder sentir tristeza y añoranza.

En un niño normal los sentimientos depresivos, sus ansiedades de haber perdido el pecho o la madre también dan lugar a sentimientos de reparación, a sentimientos de que lo que se ha destruido mediante el odio se puede restaurar mediante el amor, y que el estado de tener un bueno objeto interno puede volver otra vez, mucho menos idealizado que antes porque es un objeto que lo había frustrado a uno, es un objeto que había sido atacado; es un objeto que necesitaba una restauración y por tanto adquiría distintas características (Klein, 1957). Una vez que el niño superó la posición esquizo-paranoide y alcanzó la posición depresiva posee más integración; el superyó se vuelve más realista, promueve la reparación, la restauración, y el recuperar un buen estado de las cosas.

La frustración y las circunstancias desdichadas sin duda despiertan la envidia y el odio en cada persona a lo largo de su vida, pero la fuerza de estas 


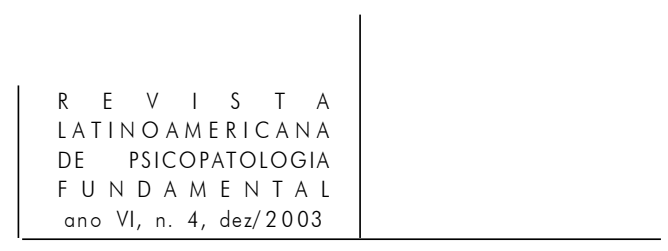

emociones y el modo de enfrentarlas varía de manera considerable. Esta es una de las numerosas razones por las cuales la capacidad de gozar, ligada al sentimiento de gratitud y al superyó, por la bondad recibida, difiere grandemente en los distintos individuos.

Las personas que aman y se sienten amadas por sus objetos internalizados nunca se sienten solos, sea cual sea su circunstancia, y conservan siempre la estima y el respeto por sí mismo y por los demás (Coderch, 1990).

Existe un ininterrumpido proceso de introyección y proyección, gracias al cual las imagos van perdiendo sus rasgos terroríficos que se originan por la proyección al exterior de pulsiones agresivas y destructivas, de manera que van asimilándose y acercándose cada vez más a las imágenes reales de los padres. Si el objeto bueno está disponible y es claro para el niño la identificación con él se fortalece y la capacidad de amar se desarrolla apoyándose en los impulsos constructivos y en la gratitud.

En este sentido la identificación proyectiva es proyectar lo agradable de uno mismo introduciéndolo en el objeto ya que no sólo se utiliza para dominar y controlar al objeto y a los demás depositando en su interior partes del propio self para, desde allí no solamente controlar, sino también para establecer una forma de comunicación. Antes de la comunicación verbal, el bebé hace llegar a la madre sus sentimientos, fantasías y necesidades a través de la identificación proyectiva, quien le devuelve sus ansiedades y temores en forma mitigada y tolerable para el bebé, y también el amor.

Las experiencias de amor y satisfacción empiezan a predominar y estos sentimientos, que forman parte de la ansiedad depresiva, estimulan la tendencia a reparar al objeto, esto es, compensar el mal que se le pudo haber ocasionado, devolviéndole el amor que de él se ha recibido, así como a subsanar el deterioro originado tanto en el objeto real externo como en el internalizado.

Así, la gratitud como sentimiento y acto de dar está ligada a la generosidad. Se es generoso con el objeto bienhechor ya que la riqueza interna deriva de haber asimilado a un objeto bueno, de modo que el individuo se torna capaz de compartir sus dones con otros (Klein, 1957).

En esta forma también mediante la identificación proyectiva el sujeto pone en el objeto no el odio por haber sido frustrado sino el amor, el agradecimiento por haber sido gratificado. Amor con amor se paga. En esta forma la proyección cumple con su función al servicio de la gratitud y no solo del dolor y el odio.

\section{c) Algunas operaciones defensivas}

El desarrollo del mundo interno y su funcionamiento en relación con el mundo externo concomitante a la evolución de la capacidad de representación 


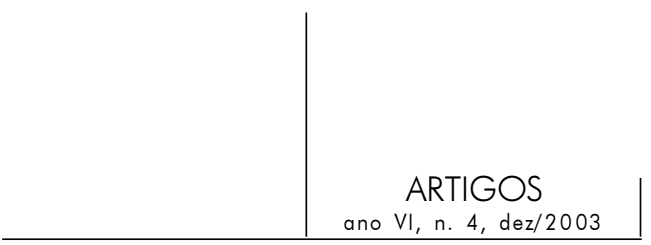

simbólica integran los siguientes componentes: la piedad, la nostalgia y la responsabilidad y, se supeditarán al genuino sentimiento de gratitud.

La piedad es una emoción compleja que surge cuando el sujeto comprueba que el objeto está sufriendo. Sin embargo, la piedad es una tendencia pasiva, lo que la torna insoportable y, como tal, no reparatoria por sí misma. Esto nos recuerda al hombre rico que, luego de escuchar los lamentos del mendigo, le ordenó al mayordomo que lo corriera, pues el pobre diablo le estaba partiendo el corazón (Eidelberg, 1965).

La piedad es una formación reactiva, por una propia necesidad narcisista pero que evoluciona hacia la empatía. Klein en su ejemplo de la obra "El niño y los sortilegios" (1929 en Petot, 1982) ilustra la piedad espontánea de un niño que siente este afecto ante un ardillita herida y a la cual le presta ayuda, y que no correspondió, en un primer momento, a ninguna necesidad del niño y sólo se basó en la identificación empática con el animal herido.

La diferencia entre la piedad y otras formaciones reactivas como lo son, la repugnancia, el orden, la limpieza etc., es que está estrechamente unida a la reparación. Petot (1982) explica que una tendencia que, para muchos autores es equivalente al término "reparación", lo es la "restitución", que sin embargo, sólo busca aplacar al perseguidor.

Conviene distinguir entre la reparación exitosa y una reparación omnipotente (maníaca o pseudoreparación) destinada al fracaso. Así, deben intervenir dos elementos: la nostalgia (con un subsecuente decremento de narcisismo) y la responsabilidad (que contiene a las otras dos: la piedad y la nostalgia). La gratitud como expresión de la capacidad reparatoria, entonces, contendrá a las tres.

Dice Amado (1985) que el individuo utiliza la inhibición y la distribución de la agresividad y también la reparación del objeto que ha sido lastimado por las pulsiones sádicas. Si fuera sólo eso, se trataría de un funcionamiento puramente utilitario del espíritu. La propia Klein (1950) admite que la reparación aspira a hacer hermoso y perfecto al objeto. Esta autora hace pues referencia a los valores estéticos, morales, sociales etc. El yo cree que el deseo de reparación del objeto atestigua el origen de la pulsión, que conserva la creencia de poder volver a crear por si mismo aquello por lo que ha sido creado. Esta ilusión es la defensa maníaca. En cambio, una reparación es sincera cuando sigue la senda de un acto creador. Entonces se trata de sublimación.

Así la nostalgia es una actitud, por tanto es una evocación del objeto. Implica el dolor de la separación y su objetivo es el acortar la distancia con el objeto. Al igual que la piedad, la nostalgia por sí misma no es reparatoria. No necesariamente se debe dar al objeto algo. De hecho, éste puede ignorar que el sujeto siente piedad o nostalgia. 




La responsabilidad es una conducta, que representa la síntesis en el acto de la dinámica de la persona suscitada por el enfrentamiento con la realidad. Comprende, como ya se mencionó, a la piedad y a la nostalgia, e implicaría un elevado sentimiento de empatía y la capacidad de dar. Sin embargo, aún con su carácter reparatorio, busca apaciguar los sentimientos de culpa y también reciprocidad, en parte, demanda un comportamiento responsable ante sus necesidades, y por otro lado, demanda un reconocimiento de la sociedad, apegándose al conjunto de ideales colectivos.

González Núñez (1987, p. 22) menciona que:

... la sublimación es un proceso mediante el cual la energía asociada a contenidos reprimidos se libera y se dirige a metas constructivas y reconstructivas socialmente aceptadas para el yo, que generalmente reconoce su epigénesis en la edad de la latencia. El concepto de sublimación generalmente no se involucra en la relación de objeto, pero a este proceso si lo ubicamos desde el punto de vista metapsicológico, podemos pensar en procesos sublimatorios desde la fase oral en la medida que el yo posee raíces innatas (Hartmann, 1969), con energía propia que pone al servicio de la supervivencia y desarrollo adaptativo del mundo interno al mundo externo y viceversa. Esa energía del yo, puesta al servicio de la adaptación tiene fines constructivos y reconstructivos para el propio sujeto y sus objetos. Si bien es cierto que se consolida y se practica en la edad de la latencia, sí podemos inferir que su génesis es anterior a esa edad.

Hartmann (1984) utilizó el término neutralización para describir la energía que utiliza la sublimación tanto con respecto al impulso sexual como al impulso agresivo. La energía neutralizada puesta así a disposición del yo puede explicar la autonomía secundaria de algunas de sus funciones. El autor aclara que no toda la energía neutralizada deriva de los impulsos, parte de ella es primaria y autónoma, así en la gratitud está implicada la energía neutralizada.

\section{La culpa como origen de la gratitud}

La condición previa para el desarrollo de las tendencias reparadoras y de las sublimaciones es que la presión del superyó disminuya y éstas las elabore el yo en forma de sentimiento de culpa. En el desarrollo ontogénico la superación de tendencias hostiles se encuentra en condiciones de superarlas por medio de la piedad y de la empatía, espera amistad y amor de los objetos reales introyectados y puede entonces estimar a los objetos reales en su capacidad de amor y en su bondad efectivas. "Cuando el niño siente piedad por la ardilla herida y la ayuda, 


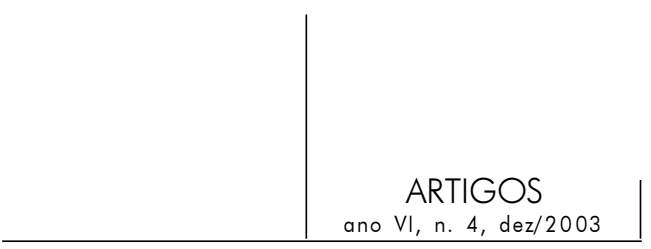

el mundo hostil se transforma en amistoso. El niño ha aprendido a amar y cree en el amor" (Klein, 1931 en Petot, 1982).

En la segunda mitad del primer año de vida el bebé experimenta, en su mundo interno, tendencias reparadoras que alcanzan sus objetivos entonces la culpa y el temor de haber destruido al objeto disminuyen y se establecen en el interior del self elementos de identificación necesarios para la formación del yo que actúan como una fuente constante de apoyo, ayuda y seguridad.

Para Winnicott (1979) idealmente existe un círculo benigno que se caracteriza por: la experiencia instintiva; la aceptación de la responsabilidad que se denomina culpa; una elaboración de la misma y un auténtico gesto de restitución. Esto puede convertirse en un círculo maligno si algo sale mal, en cuyo caso tendrá lugar una anulación de la capacidad de sentir culpa.

Klein (1950) relacionó la capacidad de sentir culpa con la posición depresiva. Para ella el pecho de la madre y todo lo que el pecho y la leche han llegado a representar en la mente del niño son: amor, bondad, seguridad. El niño siente, cuando no ha sido satisfecho, que lo ha perdido y supone que esta pérdida es el resultado de su incontrolable voracidad y de sus propias fantasías e impulsos destructivos contra el pecho de la madre. El círculo de los objetos atacados se amplía (al padre, a los hermanos). Comenta Grinberg (1983) que estos sentimientos depresivos son la fuente más profunda de los conflictos dolorosos de la situación edípica.

Lo fundamental de la posición depresiva es el sentimiento de pena por haber destruido, en la fantasía, a sus objetos y la ansiedad por preservarlos, con sus defensas correspondientes. El niño reconoce ahora al objeto total: siente que la mano que lo acaricia, la cara que le sonríe, la voz que lo calma, el pecho que no da leche, la cara que no sonríe pertenecen a la mamá, por lo que la tiene escindida: la que sí lo satisface y la que no lo satisface. El niño siente culpa cuando ataca principalmente a la que si lo satisface.

Cuando se piensa perdido al objeto amado implica una dependencia que se transforma en un incentivo para lograr la reparación y la conservación del objeto. Cada avance en el proceso de duelo da por resultado la profundización de la relación del individuo con sus objetos internos, la felicidad de reconquistarlos, después de haber sentido su pérdida.

El temor de haber lastimado y deteriorado, con la propia destructividad, al objeto necesitado y amado, origina lo que Klein (1957) denomina culpa depresiva. Las experiencias amorosas y gratificantes en relación con los padres son internalizadas y modifican las imagos hostiles y perseguidoras (culpa persecutoria), por lo tanto, en lugar del temor a ser agredido por ellos, surge la pena y el duelo por haber deteriorado al objeto $y$, como consecuencia, aparece la culpa depresiva. La elaboración de la culpa depresiva origina la gratitud. 


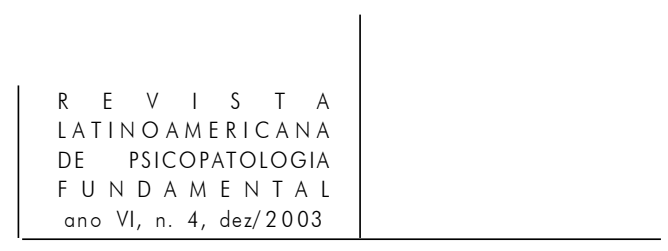

\section{La gratitud y otras cualidades}

Señalar una lógica evolutiva en valores universales tales como la fe, la esperanza y la libertad, no significa reducirlos, a su vez, a sus raíces infantiles. Más bien, nos obliga a considerar cómo los poderes humanos que van surgiendo, paso a paso, están intrínsecamente asediados no sólo por graves vulnerabilidades que exigen permanentemente nuestra comprensión terapéutica, sino también por males básicos que requieren la presencia de valores reparadores de sistemas de creencias o ideologías universales basadas en esas creencias.

\begin{tabular}{|c|c|c|c|c|}
\hline \multirow[b]{4}{*}{62} & Etapa & Crisis & Cualidad & \\
\hline & A) Infancia & $\begin{array}{l}\text { Confianza } \\
\text { Básica versus } \\
\text { Desconfianza } \\
\text { Básica }\end{array}$ & $\begin{array}{l}\text { Pulsión y } \\
\text { esperanza }\end{array}$ & $\begin{array}{l}\text { La esperanza es la confianza que se tiene de } \\
\text { recibir algo. Sus rudimentos descansan en el } \\
\text { primer encuentro que garantiza la comida y el } \\
\text { contacto cálido con la madre. }\end{array}$ \\
\hline & $\begin{array}{l}\text { B) Niñez } \\
\text { temprana }\end{array}$ & $\begin{array}{l}\text { Autonomía } \\
\text { versus } \\
\text { Vergüenza }\end{array}$ & $\begin{array}{l}\text { Autocontrol } \\
\text { y fuerza de } \\
\text { voluntad }\end{array}$ & $\begin{array}{l}\text { La voluntad es la fuerza yoica de determinarse } \\
\text { a algo, de querer, que entra en crisis por lo que } \\
\text { se desea en contraposición de las necesidades } \\
\text { de los demás. }\end{array}$ \\
\hline & $\begin{array}{l}\text { C) Edad del } \\
\text { juego }\end{array}$ & $\begin{array}{l}\text { Iniciativa } \\
\text { versus culpa }\end{array}$ & $\begin{array}{l}\text { Dirección, } \\
\text { propósito y } \\
\text { finalidad }\end{array}$ & $\begin{array}{l}\text { La finalidad es la intención, el fin con que se } \\
\text { hace algo. Es tener el propósito de ver los obje- } \\
\text { tivos del actuar. }\end{array}$ \\
\hline & $\begin{array}{l}\text { D) Edad } \\
\text { escolar }\end{array}$ & $\begin{array}{l}\text { Industria } \\
\text { versus } \\
\text { inferioridad }\end{array}$ & $\begin{array}{l}\text { Método, } \\
\text { capacidad y } \\
\text { competencia }\end{array}$ & $\begin{array}{l}\text { La competencia es la capacidad de aprender a } \\
\text { aprender que da la posibilidad de sentirse apto } \\
\text { para algo, cualidad que se conservará para el } \\
\text { resto de la vida. En esta etapa se superan los } \\
\text { sentimientos de inferioridad por incapacidad. }\end{array}$ \\
\hline & E) Adolescencia & $\begin{array}{l}\text { Identidad } \\
\text { versus } \\
\text { confusión de } \\
\text { identidad }\end{array}$ & $\begin{array}{l}\text { Devoción y } \\
\text { fidelidad }\end{array}$ & $\begin{array}{l}\text { La fidelidad es la constancia en el cariño, es la } \\
\text { adherencia a un objeto sin sentir peligro a la } \\
\text { identidad y dada esa adherencia se tiene la } \\
\text { capacidad de ser fiel. }\end{array}$ \\
\hline & F) Juventud & $\begin{array}{l}\text { Intimidad } \\
\text { versus } \\
\text { aislamiento }\end{array}$ & $\begin{array}{l}\text { Afiliación y } \\
\text { amor }\end{array}$ & $\begin{array}{l}\text { Después de poseer la devoción hacia un objeto } \\
\text { al cual se le tiene fidelidad se puede ampliar a } \\
\text { instituciones y personas a las cuales se les } \\
\text { puede amar. }\end{array}$ \\
\hline & G) Adultez & $\begin{array}{l}\text { Generatividad } \\
\text { versus } \\
\text { estancamiento }\end{array}$ & $\begin{array}{l}\text { Producción, } \\
\text { cuidado y } \\
\text { generosidad }\end{array}$ & $\begin{array}{l}\text { En la época adulta, después de haber asegura- } \\
\text { do los sentimientos de filiación y amor se pue- } \\
\text { de crear para sí mismo y compartir con los } \\
\text { demás con generosidad. En este momento se } \\
\text { ve claramente la capacidad de gratitud. }\end{array}$ \\
\hline & H) Vejez & $\begin{array}{l}\text { Integridad } \\
\text { versus } \\
\text { desesperanza }\end{array}$ & $\begin{array}{l}\text { Renuncia- } \\
\text { miento y } \\
\text { sabiduría }\end{array}$ & $\begin{array}{l}\text { Después de ser generoso, ya en la vejez, se } \\
\text { adquiere el renunciamiento a los bienes que } \\
\text { se han creado en beneficio de los objetos } \\
\text { amados: directos, desplazados o sublimados. }\end{array}$ \\
\hline
\end{tabular}




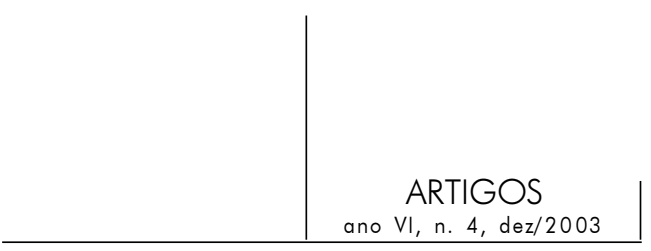

En este cuadro de cualidades Erikson no toma en cuenta la gratitud, sólo como generosidad. Sin embargo es importante señalar que, siguiendo el esquema anterior, estamos hablando de la primera etapa de confianza básica versus desconfianza básica, en la que el niño está capacitado para agradecer a la madre el bienestar que le proporciona, que lo hace sentir seguro, esto es, la gratitud nace en la gratificación de la primera carencia.

\section{La actitud de dar y tomar}

Fairbairn (1978) afirmó que la libido es por excelencia buscadora de objetos y no el placer o la descarga tal como lo planteó Freud. Existen modos de dependencia hacia el objeto que determinan el nivel de funcionamiento mental para la salud o la enfermedad y también distancias adecuadas que no le provoquen angustia ya que si el objeto está o más cerca o más lejos de lo que la persona tolera, esa cercanía o esa lejanía le producirá angustia.

Para Fairbairn (ibid.), todo el curso del desarrollo libidinoso consiste y depende de hasta que punto son incorporados los objetos. Para él los impulsos no pueden considerarse como separados de las estructuras endopsíquicas a las que dan energía y de las relaciones de objeto que permiten establecer dichas estructuras.

Este teórico plantea tres etapas de desarrollo:

\begin{tabular}{||l|l|l|l|}
\hline $\begin{array}{l}\text { I. Etapa de dependencia } \\
\text { infantil: actitud de tomar }\end{array}$ & & \\
\hline $\begin{array}{l}\text { a) Fase oral primaria: succión e } \\
\text { incorporación }\end{array}$ & Pre-ambivalente & Dependiente infantil & Actitud de tomar \\
\hline $\begin{array}{l}\text { b) Fase oral secundaria: morder } \\
\text { e incorporar }\end{array}$ & Ambivalente & Dependiente infantil & Actitud de tomar \\
\hline $\begin{array}{l}\text { II. Etapa de transición: } \\
\text { dicotomía y exteriorización } \\
\text { del objeto }\end{array}$ & Ambivalente & Dependiente infantil & Actitud de tomar \\
\hline $\begin{array}{l}\text { Ill. Etapa de dependencia } \\
\text { madura: actitud de dar }\end{array}$ & Post-ambivalente & Dependencia madura & Actitud de dar \\
\hline
\end{tabular}

En la maduración, al pasar de la fase ambivalente a la post-ambivalente, de la dependencia infantil a la dependencia madura, del tomar al dar se vuelve un proceso de agradecimiento y gratitud, esto es la actitud de tomar se vuelve una 


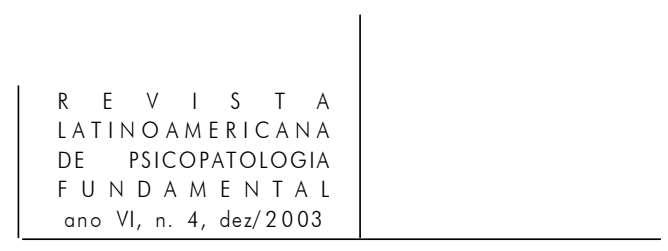

actitud de dar. Igualmente cuando el objeto se escinde en objeto bueno y malo siempre se guarda gratitud hacia la parte aceptada del mismo.

Así, el rasgo más característico de la etapa de dependencia infantil es la identificación primaria con el objeto, y su dependencia inmadura caracterizada por su actitud de tomar.

La etapa de transición comienza cuando la ambivalencia de la fase oral empieza a dar lugar a la dicotomía del objeto. Esta dicotomía es el proceso mediante el cual el objeto primario hacia el que se dirige el amor y el odio es escindido en la parte aceptada, hacia el que se dirige el amor, y en la parte rechazada, hacia el que se dirige el odio.

El desarrollo de las relaciones de objeto es un proceso por el cual la dependencia infantil del objeto da lugar, en forma gradual a la dependencia madura del mismo, y este proceso se caracteriza: a) por el abandono progresivo de una relación de objeto primaria, basada en la identificación y b) por la adopción gradual de una relación de objeto basada en la diferenciación en cuanto al objeto.

Para Fairbairn (1978) el proceso de diferenciación del objeto se hace particularmente importante por el hecho de que la dependencia infantil se caracteriza no solo por la identificación, sino por la actitud de incorporación oral.

La mayor necesidad de un niño consiste en obtener la seguridad decisiva, de que es amado por sus padres y de que éstos aceptan su amor. De no ser así, se da el trauma. Precisamente, para renunciar a la dependencia infantil, necesita de tal evidencia y, así, puede depender sin peligro de los objetos reales.

Posteriormente, en la segunda etapa, el conflicto está, entre una necesidad de desarrollo para lograr una actitud de dependencia madura con el objeto y, por otra parte, la insistencia regresiva a abandonar la actitud de dependencia infantil con dicho objeto.

Para concluir, cuanto más madura sea una relación menos caracterizada estará por la identificación primaria (fracaso de la diferenciación con el objeto) y mejor tolerará la separación de éste. Cuando la identificación persiste, a costa de la diferenciación, se introducirá en la actitud del individuo hacia sus objetos, un marcado elemento compulsivo.

\section{Envidia y gratitud}

En su libro Envidia y gratitud, Klein 1957, describe la envidia y la gratitud como dos factores dinámicos que intervienen en el psiquismo desde el nacimiento y determinan en gran parte las relaciones de objeto. Para esta autora la envidia proviene del propio sujeto, no está ligada al objeto externo que decepciona o 




frustra, y su finalidad es atacar lo que el objeto tiene como aspectos buenos, protectores y nutricios.

La envidia implica una comparación de sí mismo con otro que si posee algo, que en primera instancia es la madre, de aquí que contenga un carácter proyectivo, la madre posee un pecho lleno de leche, el niño posee un estómago vacío. En el momento en que esa carencia natural externa se convierte en personal interna, se estará desfigurando su significado y será pues estudio de la psicopatología; por lo tanto llegamos a la conclusión de que aquello que se envidia debe ser un bien, ya sea material o emocional. Se define a la envidia como: "El sentimiento enojoso contra otra persona que posee o goza de algo deseable, siendo el impulso envidioso el de quitárselo o dañarlo".

Se habla de la gratitud como uno de los sentimientos más importantes derivados de la capacidad para amar, el cual surge en las épocas más tempranas de la infancia junto con otras emociones y actitudes. Para que un niño sea agradecido debe aceptarse a sí mismo, reconocer en él la bondad, para luego reconocerla en los otros. Todo lo anterior le da al niño una riqueza interna, porque ha introyectado un mundo de beneficios que le crea una sensación de enriquecimiento, el cual, a su vez, le permite ser generoso.

La gratitud deriva de la temprana intimidad con la madre en una etapa preverbal en donde la capacidad de gozar plenamente del pecho constituye el fundamento para la experimentación de placer. Más adelante los aspectos gratificantes del objeto se han introyectado satisfactoriamente y con seguridad. Aquí el niño sabe que tiene un don inapreciable que quiere conservar y al cual le está agradecido. Si estos momentos no son interferidos por una envidia excesiva, el niño podrá en un futuro, creer en figuras buenas e identificarse con ellas.

\section{Conclusión}

De acuerdo con lo anterior, podemos ennumerar lo siguiente para hablar de gratitud:

a) Significa agradecimiento por un bien recibido.

b) Dicho bien solo puede obtenerse de otra persona que es el bienhechor.

c) Aparece en el niño desde su temprana relación con la madre.

d) Se deriva de la capacidad de amar.

e) Para que el niño la posea debe primero aceptarse a sí mismo y haber experimentado la gratificación.

f) La gratitud genera sentimiento de enriquecimiento y generosidad.

g) No se presenta si aparece la envidia. 


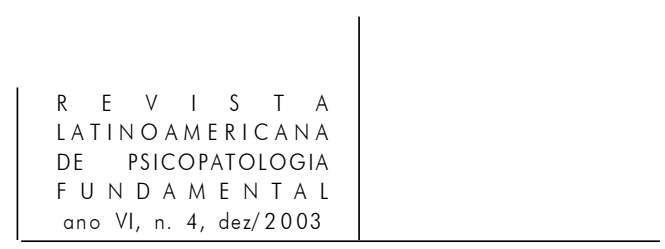

h) Presenta dos etapas de aparición:

- Primera relación gratificante con el pecho materno.

- Introyección de los aspectos gratificantes del objeto que determinarán las pautas de conducta adulta.

i) La gratitud evita a toda costa la destrucción del bien ajeno.

j) La gratitud proporciona plena capacidad de goce.

k) Este sentimiento aparecerá si se establece la confianza básica.

1) La gratitud reconoce la grandeza del don.

Cuando existe gratitud en la persona, porque no en todos los individuos existe por igual, el pronóstico de cura o de cambio interno se condiciona al mismo, esto es, a mayor gratitud mejor pronóstico, a menor gratitud peor pronóstico. Por lo tanto es importante fomentar la gratitud en el paciente, ya que ésta se puede incrementar y con esto favorecer la felicidad de la persona y la evitación de dolores emocionales innecesarios. El terapeuta debe buscar la gratitud hacia los objetos reales del paciente, no tanto hacia él. La actitud e intención de agradecer y reparar hace progresar la integración del yo. Así el paciente, al agradecer a sus objetos, crece y se restaura a sí mismo, incrementando la confianza y el propio autoaprecio.

\section{Referencias}

Amado, G. Fundamentos de la psicopatología. Locura, enfermedad mental y psiquiatría según una ontología psicoanalítica. Barcelona: Gedisa, 1985.

CoDerch, J. Teoría y técnica de la psicoterapia psicoanalítica. Barcelona: Editorial Herder, 1990.

Eidelberg, L. Psicología de la violación. Buenos Aires: Hormé, 1965.

ERIKSON, E. Infancia y sociedad. Buenos Aires: Paidós, 1963.

Fairbairn, R. Estudio Psicoanalítico de la Personalidad. Argentina: Hormé, 1978.

GonZÁlez NúÑEZ, J. J. Psicoterapia, reparación y contratransferencia. Alêtheia. Instituto de Investigación en Psicología Clínica y Social, S. A. n. 8, p. 16-25, 1987.

Grinberg, L. Culpa y depresión. Un estudio psicoanalítico. Madrid: Alianza Universidad Textos Madrid, 1983.

Hartmann, H. La psicología del yo y el problema de la adaptación. México: Paidós, 1984.

Klein, M. El duelo y su relación con los estados maníaco-depresivos. Obras Completas. España: Paidós, 1950.

KLein, M. La técnica psicoanalítica del juego: su historia y significado. Obras Completas. España: Paidós, 1955. 


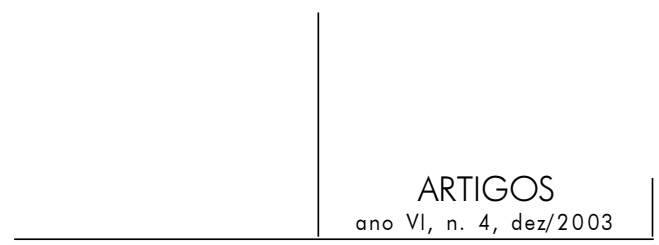

KLeIn, M. Envidia y gratitud. Obras Completas. España: Paidós, 1957.

Рetot, J. Melanie Klein. Primeros descubrimientos y primer sistema (1919-1932). Buenos Aires: Paidós, 1982.

RAPAPORT, D. Nuevas Aportaciones a la teoría y técnica psicoanalítica. México: Editorial Pax, 1967.

SEgAL, H. Introducción a la obra de Melanie Klein. México: Paidós, 1992.

WinNicotT, D. (1979). La agresión en relación con el desarrollo emocional. In: Escritos de pediatría Mahler y psicoanálisis. Barcelona: Laia, 1986.

\section{Resumos}

Neste artigo conceitua-se a gratidão como um afeto cuja definição é a capacidade de o indivíduo agradecer, poder reconhecer os benefícios recebidos de um benfeitor. Discute-se a epigênese e evolução da gratidão junto com outros processos psíquicos como: gratidão e defesa; gratidão e sentimentos de culpa; gratidão e outras qualidades humanas; gratidão e inveja, para concluir com qual é a função da gratidão no processo de psicoterapia psicanalítica.

Palavras-chave: Gratidão, defesas, sentimentos de culpa, inveja

Dans cet article, la gratitude est conceptualisée comme un affect dont la définition est la capacité de l'individu à remercier, à pouvoir reconnaître les bénéfices reçus d'un bienfaiteur. On y discute l'épigenèse et l'évolution de la gratitude conjointement avec d'autres processus psychiques tels que la gratitude et la défense, la gratitude et les sentiments de culpabilité, la gratitude et d'autres qualités humaines, la gratitude et l'envie, et pour conclure quelle est la fonction de la gratitude dans le processus de psychothérapie psychanalytique.

Mots clés: Gratitude, défenses, sentiments de culpabilité, envie

Gratitude is conceived of here as a feeling derived from an individual's capacity for being grateful, of recognizing gifts received from a benefactor.

The epigenesis and evolution of gratitude are discussed, together with other psychic processes, such as defenses, guilt feelings, envy and other human traits. The objective is to determine the function of gratitude in the field of psychoanalytic psychotherapy.

Key words: Gratitude, defenses, guilt feelings, envy 